\title{
Coupling of spin and orbital motion of electrons in carbon nanotubes
}

Kuemmeth, Ferdinand; Ilani, S; Ralph, D C; McEuen, P L

Published in:

Nature

DOI:

doi:10.1038/nature06822

Publication date:

2008

Document version

Publisher's PDF, also known as Version of record

Citation for published version (APA):

Kuemmeth, F., Ilani, S., Ralph, D. C., \& McEuen, P. L. (2008). Coupling of spin and orbital motion of electrons in carbon nanotubes. Nature, 452(7186), 448-452. https://doi.org/doi:10.1038/nature06822 


\title{
Coupling of spin and orbital motion of electrons in carbon nanotubes
}

\author{
F. Kuemmeth ${ }^{1 *}$, S. Ilani ${ }^{1 *}$, D. C. Ralph ${ }^{1}$ \& P. L. McEuen ${ }^{1}$
}

Electrons in atoms possess both spin and orbital degrees of freedom. In non-relativistic quantum mechanics, these are independent, resulting in large degeneracies in atomic spectra. However, relativistic effects couple the spin and orbital motion, leading to the well-known fine structure in their spectra. The electronic states in defect-free carbon nanotubes are widely believed to be four-fold degenerate ${ }^{1-10}$, owing to independent spin and orbital symmetries, and also to possess electron-hole symmetry ${ }^{11}$. Here we report measurements demonstrating that in clean nanotubes the spin and orbital motion of electrons are coupled, thereby breaking all of these symmetries. This spin-orbit coupling is directly observed as a splitting of the four-fold degeneracy of a single electron in ultra-clean quantum dots. The coupling favours parallel alignment of the orbital and spin magnetic moments for electrons and antiparallel alignment for holes. Our measurements are consistent with recent theories ${ }^{12,13}$ that predict the existence of spin-orbit coupling in curved graphene and describe it as a spindependent topological phase in nanotubes. Our findings have important implications for spin-based applications in carbonbased systems, entailing new design principles for the realization of quantum bits (qubits) in nanotubes and providing a mechanism for all-electrical control of spins ${ }^{14}$ in nanotubes.

Carbon-based systems are promising candidates for spin-based applications such as spin-qubits ${ }^{14-19}$ and spintronics ${ }^{20-23}$ as they are believed to have exceptionally long spin coherence times because of weak spin-orbit interactions and the absence of nuclear spin in the ${ }^{12} \mathrm{C}$ atom. Carbon nanotubes may have a particularly interesting role in this context because in addition to spin they offer a unique twofold orbital degree of freedom that can also be used for quantum manipulation. The latter arises from the two equivalent dispersion cones $\left(K\right.$ and $K^{\prime}$ ) in graphene, which lead to doubly degenerate electronic orbits that encircle the nanotube circumference in a clockwise and anticlockwise fashion ${ }^{24}$ (Fig. 1a). Together, the two-fold spin degeneracy and two-fold orbital degeneracy are generally assumed to yield a four-fold-degenerate electronic energy spectrum in clean nanotubes. Understanding the fundamental symmetries of this spectrum is at the heart of successful manipulation of these quantum degrees of freedom.

A powerful way to probe the symmetries is by confining the carriers to a quantum dot and applying a magnetic field $B_{\|}$parallel to the tube axis ${ }^{4,5,8,10,24,25}$. The confinement creates bound states and the field interrogates their nature by coupling independently to their spin and orbital moments. In the absence of spin-orbit coupling, such a measurement should yield for a defect-free nanotube the energy spectrum shown in Fig. 1b. At $B_{\|}=0$ the nanotube spectrum should be four-fold degenerate. With increasing $B_{\|}$the spectrum splits into pairs of anticlockwise and clockwise states (going down and up in energy respectively), each pair having a smaller internal spin splitting.
Indications of approximate four-fold degeneracy have been observed in high-field measurements of electron addition spectra ${ }^{2-10}$ and inelastic cotunnelling ${ }^{4,10}$ in nanotube quantum dots. However, in previous experiments disorder-induced splitting of the orbital degeneracy and electron-electron interactions in multi-electron quantum dots have masked the intrinsic symmetries at low energies.

In this work we directly measure the intrinsic electronic spectrum by studying a single charge carrier, an electron or a hole, in an ultraclean carbon nanotube quantum dot. Remarkably, we find that the expected four-fold symmetry and electron-hole symmetry are broken by spin-orbit coupling, demonstrating that the spin and orbital motion in nanotubes are not independent degrees of freedom. The observed spin-orbit coupling further determines the filling order in the many-electron ground states, giving states quite different from models based purely on electron-electron interactions.

The geometry of our devices is shown in Fig. 1c. A single smallbandgap nanotube is contacted by source and drain electrodes, and is gated from below by two gates (see Methods). When biased, these gates shift the local Fermi energy in the nanotube, thereby accumulating electrons or holes. In this work we use two independent gates to create a quantum dot that is localized above either the left or the right gate electrode. This is achieved by choosing appropriate combinations of gate voltages that pin the Fermi energy inside the gap on one side of the device while adding carriers to the other side (Fig. 1c). Measurement of the linear conductance, $G=\mathrm{d} I / \mathrm{d} V_{\text {sd }}$, through such a dot (Fig. 1e) shows Coulomb blockade peaks that correspond to the addition of individual carriers to the dot, and allows us to identify the first electron and first hole in the dot (see Supplementary Information for details). Having a single carrier in the dot enables us to study single-particle levels in the absence of electron-electron interactions, and thus to identify unambiguously the presence of spinorbit coupling. The results reported here were observed in two independent devices and below we present data from one of them.

We probe the quantum states of the nanotube using tunnelling spectroscopy. The differential conductance through the dot, $G=$ $\mathrm{d} I / \mathrm{d} V_{\text {sd }}$, is measured as a function of gate voltage, $V_{\mathrm{g}}$, and sourcedrain bias, $V_{\mathrm{sd}}$, as the first electron is added to the dot. Figure $2 \mathrm{a}$ shows a typical measurement taken at $B_{\|}=300 \mathrm{mT}$. The transition between the Coulomb blockade regions of zero and one electron features distinct resonances that correspond to the ground state $(\alpha)$ as well as the excited states $(\beta, \gamma, \delta)$ of the first electron. Their energies can be obtained from a line cut at constant $V_{\text {sd }}$ (Fig. 2b), by converting the gate voltages into energies (see Methods).

The magnetic field dependence of the one-electron states $\alpha, \beta, \gamma$ and $\delta$ is measured by taking $V_{\mathrm{g}}$ traces such as in Fig. 2b for different values of $B_{\|}$. This is shown in Fig. $2 \mathrm{c}$, where we plot $\mathrm{d} I / \mathrm{d} V_{\text {sd }}$ as a function of $V_{\mathrm{g}}$ and $B_{\|}$. The energies of the states $\alpha$ and $\beta$ decrease with increasing $B_{\|}$, so we identify them as anticlockwise orbital states. The 
states $\gamma$ and $\delta$ increase in energy with increasing $B_{\|}$and are thus identified as clockwise orbital states. From the slopes of these resonances with respect to magnetic field we extract an orbital moment of $\mu_{\mathrm{orb}}=1.55 \mathrm{meVT}^{-1}$ and estimate the nanotube diameter to be $d \approx 5 \mathrm{~nm}$ (ref. 24).

A striking difference is observed when we compare the measured excitation spectrum with that predicted in Fig. 1b: at zero magnetic field the four states in our measurement are not degenerate but rather split into two pairs. To identify the nature of this splitting we note that with increasing magnetic field the energy difference between the states $\alpha$ and $\beta$ increases while the difference between states $\gamma$ and $\delta$ decreases, and both differences are consistent with a $g$-factor of an electron spin (Fig. 2d). This observation allows us to identify unambiguously the spin and orbital composition of each energy level, as shown in the inset of Fig. 2c. At $B_{\|}=0$ the four-fold degeneracy is split into two Kramer doublets-the lower-energy doublet involves states with parallel alignment of orbital and spin magnetic moments, whereas the higher-energy doublet has states with antiparallel alignment. The zero-field splitting is therefore identified as a spin-orbit splitting, with a value of $\Delta_{\mathrm{SO}}=0.37 \pm 0.02 \mathrm{meV}$ (extracted from Fig. 2d).

At low fields (Fig. 2e) the intersections of states with opposite spin directions (for example, $\alpha$ and $\gamma$ ) show simple crossing, whereas states with parallel spin (for example, $\beta$ and $\gamma$ ) show avoided crossing, a signature of disorder-induced mixing between anticlockwise and clockwise orbits $\left(\Delta_{K K^{\prime}}\right)$. In previous experiments, the disorderinduced mixing was significantly larger, presumably obscuring the effects of spin-orbit coupling. In our measurements, the mixing is small, $\Delta_{K K^{\prime}} \approx 65 \mu \mathrm{eV} \ll \Delta_{\mathrm{SO}}$, probably owing to smooth electronic confinement, allowing the observation of spin-orbit effects. We further demonstrate the intrinsic nature of the effect by measuring identical excitation spectra for quantum dots formed at different locations along the same nanotube (Supplementary Fig. 1).

Next, we show that spin-orbit coupling significantly affects the many-body ground states of multiple electrons in a quantum dot.
Figure 3 a shows the magnetic field dependence of the addition energies for the $N$-electron ground states $(N=-2$ to +4$)$, obtained by measuring the linear conductance as a function of $V_{\mathrm{g}}$ and $B_{\|}$. Near zero magnetic field the sign of $\mathrm{d} V_{\mathrm{g}} / \mathrm{d} B_{\|}$changes every time an electron is added (or removed), indicating that anticlockwise and clockwise states are filled alternately. Similar addition sequences were explained in the past by repulsive electron-electron interactions driving electrons to occupy different orbits ${ }^{2-7,9,26}$ (Fig. 3b). However, in our nanotubes the underlying mechanism is entirely different. Comparing the one-electron excitation spectrum with the two-electron ground state (Fig. 3c), we see that the latter follows exactly the first excited state of the one-electron quantum dot. Specifically, both start with a clockwise slope at low fields and flip to an anticlockwise slope at the field associated with the spin-orbit splitting, $B_{\|} \approx 125 \mathrm{mT}$. Thus the two-electron ground state is explained entirely by spin-orbit coupling (Fig. 3d). Note that below $B_{||} \approx 125 \mathrm{mT}$ spin-orbit coupling favours each of the two electrons to possess parallel orbital and spin moments, forcing them into two different orbital states. Therefore, the two-electron ground state is neither the spin-triplet state predicted by the electron-interactionbased models nor a spin singlet, but rather a Slater determinant of two single-electron states each of which has parallel orbital and spin magnetic moments.

Spin-orbit effects are commonly assumed to be negligible in carbon-based systems because of the weak atomic spin-orbit splitting in carbon $\left(\Delta_{\mathrm{at}}=E\left({ }^{2} P_{3 / 2}\right)-E\left({ }^{2} P_{1 / 2}\right) \approx 8 \mathrm{meV}\right.$ ) (ref. 27) and its almost perfect suppression in flat graphene ${ }^{13}$. But recent theories have argued that spin-orbit coupling can nevertheless be significant in carbon nanotubes owing to their curvature and cylindrical topo$\log y^{12,13}$. The predicted effect is illustrated in Fig. 4a. Consider an electron with a spin moment pointing along the nanotube axis and orbiting around the nanotube circumference. The electron occupies the $p_{z}$ orbitals of the carbon atoms, which are pointing perpendicular to the nanotube surface. In the rest frame of the electron the underlying $p_{z}$ orbital revolves around the spin exactly once every rotation, a

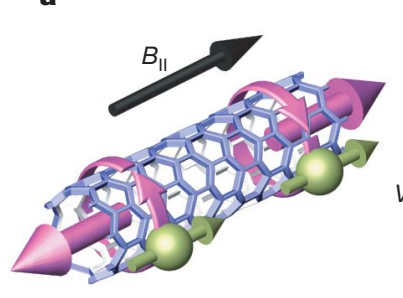

b

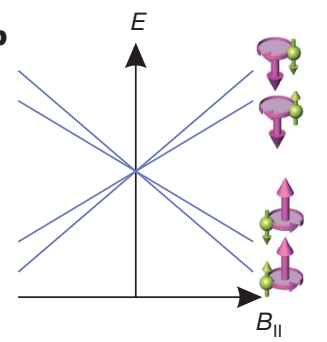

c

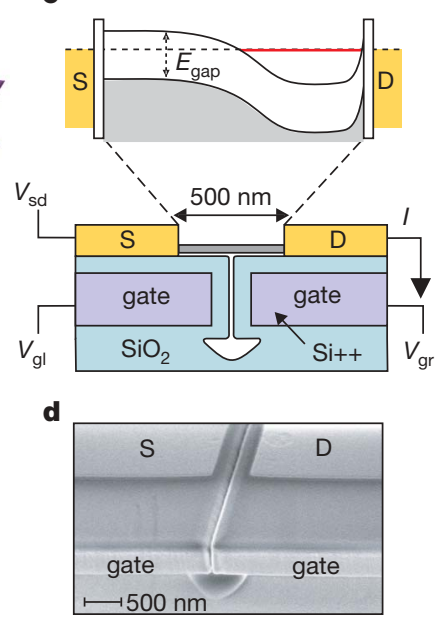

e

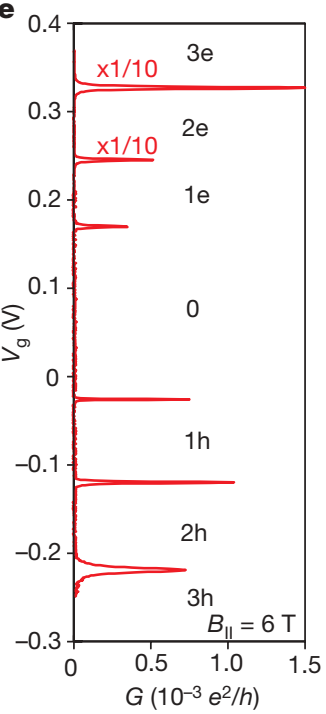

Figure 1 | Few-electron carbon nanotube quantum dot devices. a, Electrons confined in a nanotube segment have quantized energy levels, each four-fold degenerate in the absence of spin-orbit coupling and defect scattering. The purple arrow at the left (right) illustrates the current and magnetic moment arising from clockwise (anticlockwise) orbital motion around the nanotube. The green arrows indicate positive moments due to spin. $\mathbf{b}$, Expected energy splitting for a defect-free nanotube in a magnetic field $B_{\|}$parallel to the nanotube axis in the absence of spin-orbit coupling: At $B_{\|}=0 \mathrm{~T}$, all four states are degenerate. With increasing $B_{\|}$each state shifts according to its orbital and spin magnetic moments, as indicated by purple and green arrows respectively. c, Device schematic. A single nanotube makes contact to source and drain electrodes, separated by $500 \mathrm{~nm}$, and is gated from below by two gate electrodes. The two gate voltages $\left(V_{\mathrm{gl}}, V_{\mathrm{gr}}\right)$ are used to create a quantum dot localized above the right or left gate electrodes. The energy band diagram is shown for the first case. d, Scanning electron micrograph of the device, taken before nanotube growth to avoid damage to the nanotube. e, The measured linear conductance, $G=\mathrm{d} I / \mathrm{d} V_{\text {sd }}$, as function of gate voltage, $V_{\mathrm{g}}$, for a dot localized above the right gate $\left(B_{\|}=6 \mathrm{~T}\right.$, temperature $\left.T=30 \mathrm{mK}\right)$. The number of electrons or holes in the dot is indicated. The conductance of the top two peaks is scaled by $1 / 10$. 
independent of the details of the electron trajectory. In the presence of atomic spin-orbit coupling a constant phase accumulates during each rotation, which can therefore be described by a spin-dependent topological flux, $S_{\|} \phi_{\text {SO }}$, passing through the nanotube cross-section $\left(S_{\|}=+1 /-1\right.$ for spin moment parallel/antiparallel to the nanotube axis). This flux modifies the quantization condition of the wavefunction around the circumference:

$$
k_{\perp} \pi d \rightarrow k_{\perp} \pi d-2 \pi S_{\|} \phi_{\mathrm{SO}} / \phi_{0}
$$

where $k_{\perp}$ is the electron's wave vector in the circumferential direction as measured from the $K$ and $K^{\prime}$ points, $d$ is the tube diameter and $\phi_{0}$ is

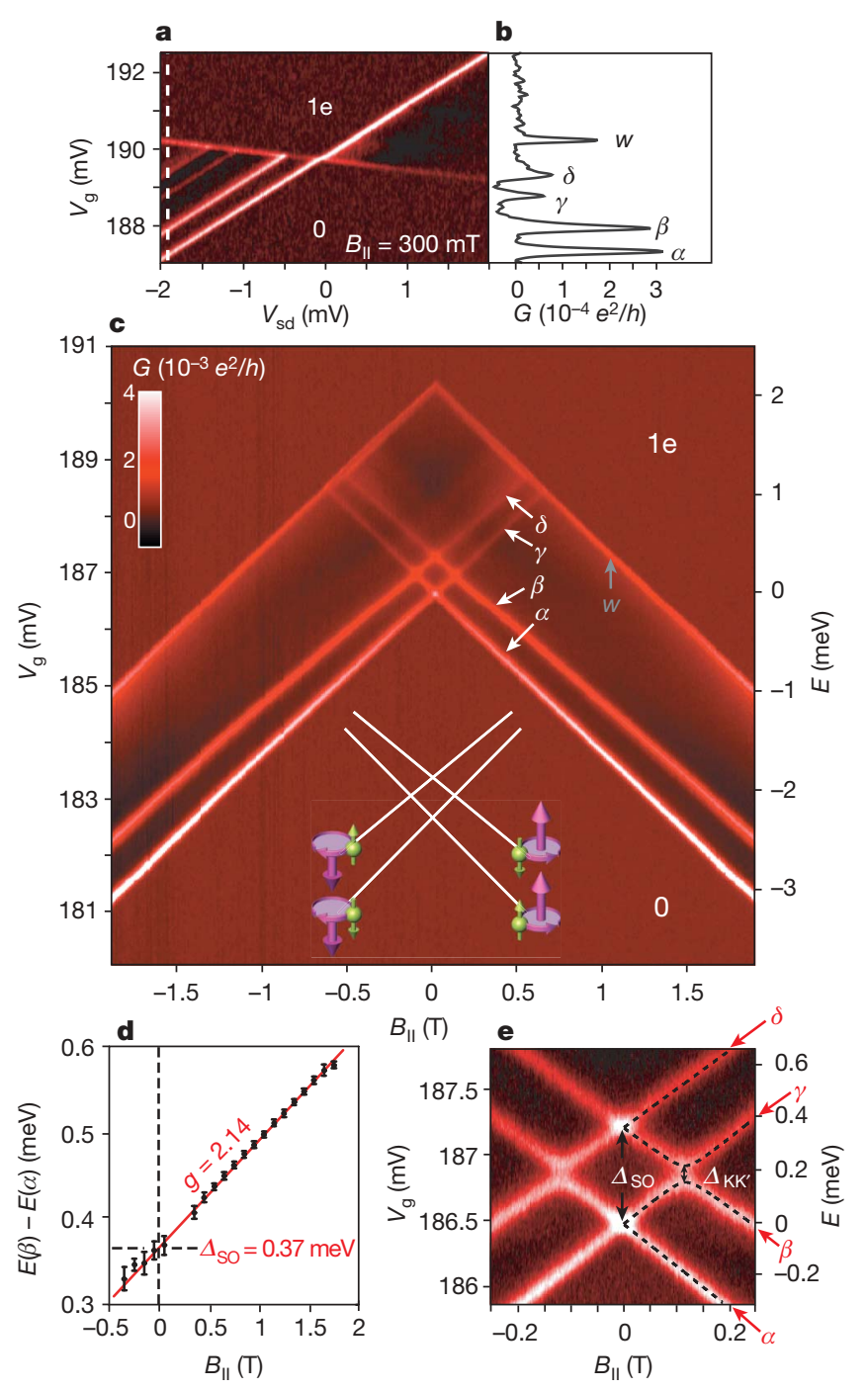

Figure 2 | Excited-state spectroscopy of a single electron in a nanotube dot. a, Differential conductance, $G=\mathrm{d} I / \mathrm{d} V_{\text {sd }}$, measured as function of gate voltage, $V_{\mathrm{g}}$, and source-drain bias, $V_{\mathrm{sd}}$, at $B_{\|}=300 \mathrm{mT}$, displaying transitions from zero to one electron in the dot. $\mathbf{b}$, A line cut at $V_{\text {sd }}=-1.9 \mathrm{mV}$ reveals four energy levels $\alpha, \beta, \gamma$ and $\delta$ as well as another peak $w$ corresponding to the edge of the one-electron Coulomb diamond. c, $G=\mathrm{d} I / \mathrm{d} V_{\text {sd }}$ as a function of $V_{\mathrm{g}}$ and $B_{\|}$at a constant bias $V_{\text {sd }}=-2 \mathrm{mV}$. The resonances $\alpha, \beta, \gamma, \delta$ and $w$ are indicated. The energy scale on the right is determined by scaling $\Delta V_{\mathrm{g}}$ with the conversion factor $\alpha=0.57$ extracted from the slopes in a. Inset: orbital and spin magnetic moments assigned to the observed states. d, Extracted energy splitting between the states $\alpha$ and $\beta$ as a function of $B_{\|}$(dots). The linear fit (red line) gives a Zeeman splitting with $g=2.14 \pm 0.1$, and a zero-field splitting of $\Delta_{\mathrm{so}}=0.37 \pm 0.02 \mathrm{meV}$ (error bars, 1 s.d.). e, Magnified view of panel c showing the zero-field splitting due to spin-orbit interaction $\left(\triangle_{\mathrm{SO}}\right)$ as well as finite-field anticrossing due to $K-K^{\prime}$ mixing $\left(\Delta_{K K^{\prime}}\right)$. Dashed lines show the calculated spectrum using $\Delta_{K K^{\prime}}=65 \mu \mathrm{eV}$. the flux quantum. According to the theory in ref. 12 the flux is given by

$$
\phi_{\mathrm{SO}}=\frac{\Delta_{\mathrm{at}}}{12 \varepsilon_{\pi \sigma}}\left(5+3 \frac{V_{\mathrm{pp}}^{\sigma}}{V_{\mathrm{pp}}^{\pi}}\right) \phi_{0} \approx 10^{-3} \phi_{0}
$$

where $\varepsilon_{\pi \sigma}$ is the energy splitting of the $\pi$ and $\sigma$ bands in graphene and $V_{\mathrm{pp}}^{\sigma}, V_{\mathrm{pp}}^{\pi}$ are the hopping elements within these bands. This flux does not depend on the geometrical properties of the nanotube such as its diameter or the shape of its cross-section, signifying its topological origin.

Figure $4 \mathrm{~b}$ illustrates the consequences of the modified quantization conditions for a small-bandgap tube at $B_{\|}=0$. Near each Dirac cone $\left(K\right.$ and $\left.K^{\prime}\right)$ there are two quantization lines for the two spin directions (dashed lines). Combining equation (1) with the linear dispersion, and including the Aharonov-Bohm flux induced by $B_{\|}$, $\phi_{\mathrm{AB}}=B_{||} \pi d^{2} / 4$, and the Zeeman spin coupling, the energies are

$$
E= \pm \hbar v_{\mathrm{F}} \sqrt{k_{\|}^{2}+k_{\perp}^{2}}-\frac{g}{2} \mu_{\mathrm{B}} S_{\|} B_{\|}, \quad k_{\perp}=k_{\perp, 0}+\frac{2}{d} \frac{\phi_{\mathrm{AB}}}{\phi_{0}}+S_{\|} \frac{2}{d} \frac{\phi_{\mathrm{SO}}}{\phi_{0}}
$$

Here $v_{\mathrm{F}}$ is the Fermi velocity, $k_{\|}$is the wave vector parallel to the nanotube axis, and $k_{\perp, 0}= \pm E_{\text {gap }} / 2 \hbar v_{\mathrm{F}}$ accounts for the small bandgap, $E_{\text {gap }}$, at zero magnetic field (the opposite signs are for the $K^{\prime}$ and $K$ points). The resulting energy spectrum is schematically shown in
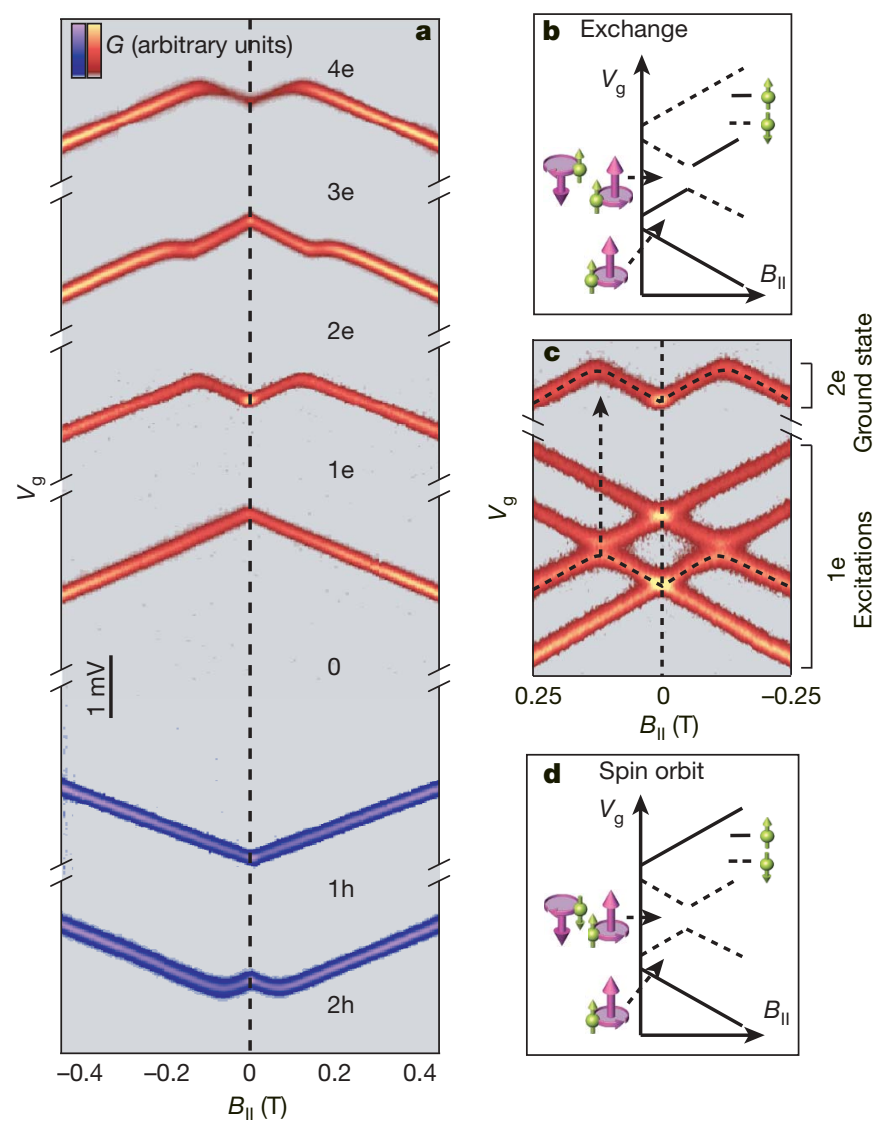

Figure 3 | The many-electron ground states and their explanation by spinorbit interaction. a, $G=\mathrm{d} I / \mathrm{d} V_{\mathrm{sd}}$, measured as a function of gate voltage, $V_{\mathrm{g}}$, and magnetic field, $B_{\|}$, showing Coulomb blockade peaks (carrier addition spectra) for the first four electrons and the first two holes (data are offset in $V_{\mathrm{g}}$ for clarity). $\mathbf{b}$, Incorrect interpretation of the addition spectrum shown in a using a model with exchange interactions between electrons. Dashed/solid lines represent addition of down/up spin moments. The two-electron ground state at low fields, indicated at the left, is a spin triplet. c, Comparison of the measured two-electron addition energy from a with the one-electron excitation spectrum from Fig. 2e. d, Schematic explanation of the data in a using electronic states with spin-orbit coupling: The two-electron ground state at low fields, indicated on the left, is neither a spin-singlet nor a spin-triplet state. 
Fig. 4c, and is in agreement with our measurements. From equation (3) we see that the spin-orbit energy splitting $\Delta_{\mathrm{SO}}=\frac{4 \hbar v_{\mathrm{F}}}{d} \frac{\phi_{\mathrm{SO}}}{\phi_{0}}$ (assuming $k_{\|}=0$ ) is inversely proportional to $d$. Using the estimated diameter of our nanotube, $d \approx 5 \mathrm{~nm}$, and the measured splitting (Fig. $2 \mathrm{~d}$ ) we obtain the value $\Delta_{\mathrm{SO}} \approx 1.9 / \mathrm{dmeV} \mathrm{nm}^{-1}$, in agreement with the predicted $^{13}$ value of $\Delta_{\mathrm{SO}} \approx 1.6 / \mathrm{d} \mathrm{meV} \mathrm{nm}^{-1}$.

An interesting prediction of the theory ${ }^{12,13,18}$ is the breaking of electron-hole symmetry. In the absence of spin-orbit interactions the low-energy spectrum of a nanotube shows electron-hole symmetry such that each allowed state has a matching state with opposite energy; that is, the spectrum is symmetric on reflection about the line $E=0$. In the presence of spin-orbit interactions and an applied magnetic field, equation (3) predicts that this symmetry is broken, as is evident from the absence of mirror symmetry around $E=0$ in the spectrum in Fig. $4 \mathrm{c}$. For $\phi_{\mathrm{SO}}>0$, the theory predicts that in the oneelectron ground state the orbital and spin magnetic moments are parallel, whereas in the one-hole ground state they are antiparallel. This result allows us to test the breaking of electron-hole symmetry experimentally.

The measured excitation spectra for the first hole in the quantum dot (Fig. 4d) clearly shows a spin-orbit splitting at $B_{\|}=0$, and a spin $g$-factor equal to that of the one-electron quantum dot (Fig. 4e).
However, in contrast to the one-electron case, here the ground state $(\alpha)$ and the first excited state $(\beta)$ converge with increasing $B_{\|}$, implying that the orbital and spin moments are aligned antiparallel in the one-hole ground state, opposite to the one-electron case. This observation qualitatively confirms the scheme in Fig. 4c. We note, however, that the spin-orbit splitting observed for the hole $\left(\Delta_{\mathrm{SO}}=\right.$ $0.21 \pm 0.01 \mathrm{meV})$ is somewhat smaller than that of the electron, a difference that is not accounted for by current theory. This might result from different confinement lengths (different $k_{\|}$in equation (3)) or different electric fields (that is, different $\left|V_{\mathrm{g}}\right|$ ) for electrons and holes, but current theories predict an effect that is too small to explain this observation.

The existence of spin-orbit coupling in carbon nanotubes invalidates several common assumptions about the nature of the electronic states in this system, such as four-fold degeneracy and electron-hole symmetry, and further leads to the existence of entangled spin and orbital multi-electron ground states. Currently, carbon-based systems are considered to be excellent candidates for spin-based applications, in part because of the belief that they have weak spin-orbit interactions. Here we have shown that this hypothesis is wrong for nanotubes. Nevertheless, rather than excluding spin-based devices in nanotubes, our findings may actually promote their feasibility, as long as new design principles are adopted for qubits and spintronic a
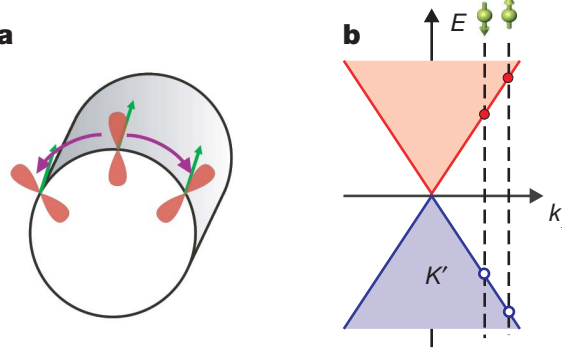

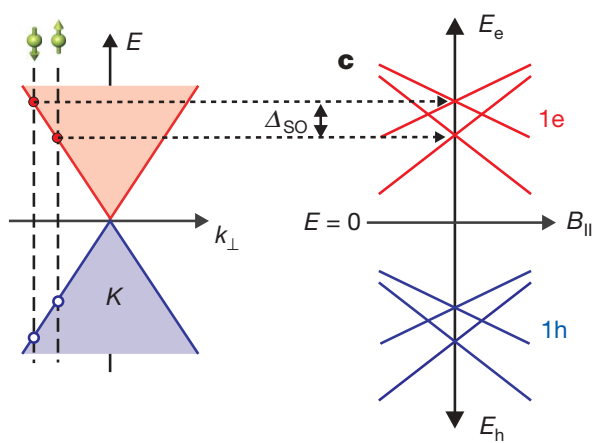

$E_{\mathrm{h}}$
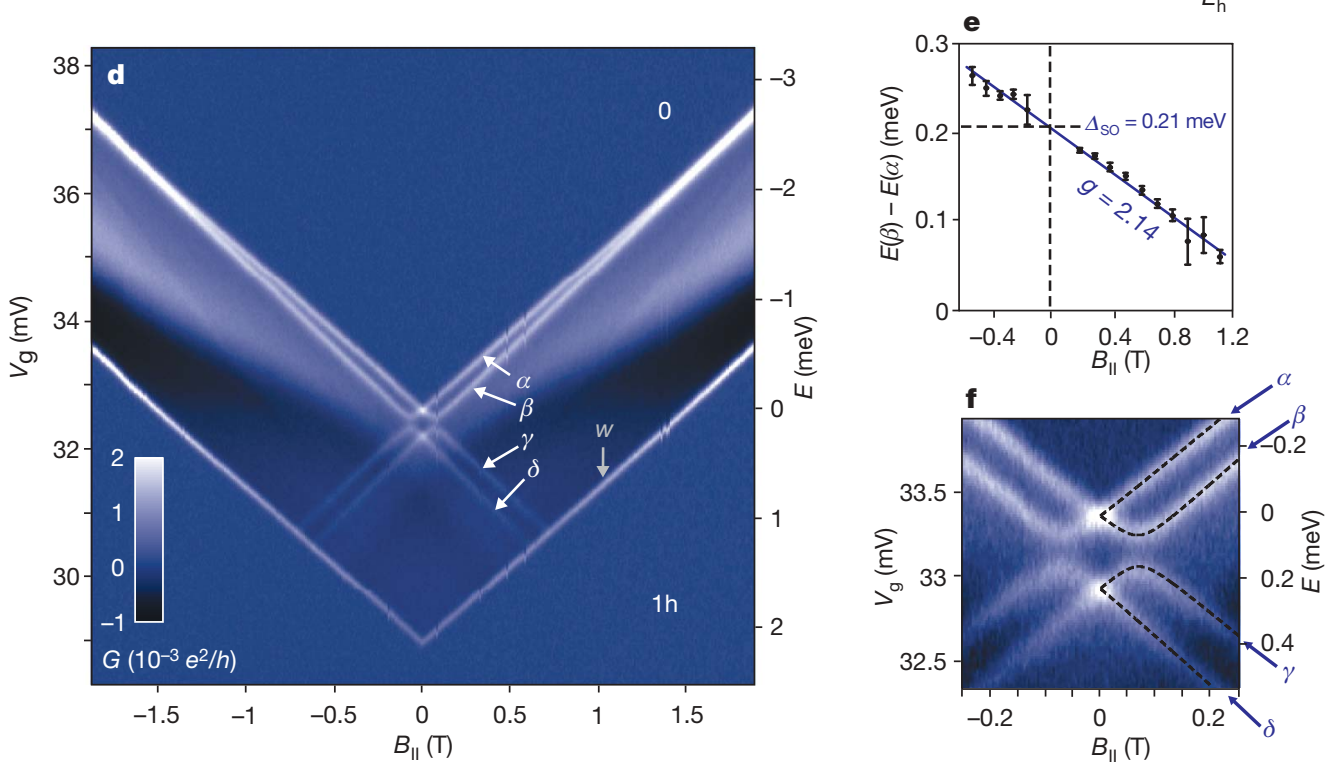

Figure 4 | Theoretical model for spin-orbit interaction in nanotubes and the energy level spectroscopy of a single hole. a, Schematic of an electron with spin parallel to the nanotube axis revolving around the nanotube circumference. The carbon $p_{z}$ orbitals (red) are perpendicular to the surface. In the rest frame of the electron, the $p_{z}$ orbital rotates around the spin. b, Allowed electron and hole energies (red dots and blue circles) at $B_{\|}=0$ for a small-bandgap nanotube with spin-orbit interaction. The states are derived by cutting the Dirac cones ( $K$ and $K^{\prime}$ ) with spin-dependent quantization lines (dashed lines). The allowed $k_{\perp}$-vectors differ for up and down electron spin moments. c, Calculated energy levels for an electron (red lines) and a hole (blue lines) as a function of $B_{\|}$. The four distinct slopes arise from the orbital and spin Zeeman shifts. d, $G=\mathrm{d} I / \mathrm{d} V_{\text {sd }}$ as a function of $V_{\mathrm{g}}$ and $B_{\|}$at a constant bias $V_{\mathrm{sd}}=-2 \mathrm{mV}$. The resonances labelled $\alpha, \beta, \gamma, \delta$ and $w$ arise from tunnelling of holes onto the dot and therefore the energy scale runs in the opposite direction to $V_{\mathrm{g}}$. The ground state $(\alpha)$ and first excited state $(\beta)$ cross at $B_{\|} \approx 1.5 \mathrm{~T}$. e, Extracted energy splitting between the states $\alpha$ and $\beta$ as a function of $B_{\|}$(dots). The linear fit (blue line) gives a Zeeman splitting with $g=2.14 \pm 0.1$, and a zero-field splitting of $\Delta_{\text {SO }}=0.21 \pm 0.01 \mathrm{meV}$ (error bars, 1 s.d.). f, Magnified view of the level crossings in $\mathbf{d}$ and a model calculation using $\Delta_{K K^{\prime}}=0.1 \mathrm{meV}$ (dashed lines). 
devices, which make use of the strong spin-orbit coupling. This coupling can provide a valuable capability that has so far been missing from carbon systems: the ability to use electrical gates to manipulate the spin degree of freedom, through its coupling to the orbital electronic wavefunction ${ }^{14}$.

\section{METHODS SUMMARY}

We fabricated devices from degenerately doped silicon-on-insulator wafers, with a $1.5-\mu \mathrm{m}$-thick device layer on top of a $2-\mu \mathrm{m}$ buried oxide. Two electrically isolated gate electrodes (Fig. 1d) were patterned from the device layer using dry etching and thermal oxidation (thickness $100 \mathrm{~nm})$. Gate contacts $(2 / 50 \mathrm{~nm}$ $\mathrm{Ti} / \mathrm{Pt})$, source and drain electrodes $(5 / 25 \mathrm{~nm} \mathrm{Cr} / \mathrm{Pt})$ and catalyst pads were patterned using electron-beam lithography. Nanotubes were grown after completing all patterning to produce clean devices ${ }^{8}$. We made measurements in a ${ }^{3} \mathrm{He} /{ }^{4} \mathrm{He}$ dilution refrigerator at base temperature $(T=30 \mathrm{mK})$, using standard lock-in techniques with small excitations (typically $4-10 \mu \mathrm{V}$ ). The electron temperature extracted from Coulomb peak widths was $100-200 \mathrm{mK}$. The conversion from gate voltage to energy is obtained from the bias dependence of the tunnelling resonances, such as in Fig. 2a, and is $\alpha=0.57$ for the first electron (Fig. 2) and $\alpha=0.58$ for the first hole (Fig. 4).

\section{Received 21 November 2007; accepted 5 February 2008.}

1. Kane, C. L. \& Mele, E. J. Size, shape, and low energy electronic structure of carbon nanotubes. Phys. Rev. Lett. 78, 1932-1935 (1997).

2. Cobden, D. H. \& Nygard, J. Shell filling in closed single-wall carbon nanotube quantum dots. Phys. Rev. Lett. 89, 046803 (2002).

3. Liang, W. J., Bockrath, M. \& Park, H. Shell filling and exchange coupling in metallic single-walled carbon nanotubes. Phys. Rev. Lett. 88, 126801 (2002).

4. Jarillo-Herrero, P. et al. Electronic transport spectroscopy of carbon nanotubes in a magnetic field. Phys. Rev. Lett. 94, 156802 (2005).

5. Jarillo-Herrero, P. et al. Orbital Kondo effect in carbon nanotubes. Nature 434, 484-488 (2005).

6. Moriyama, S. et al. Four-electron shell structures and an interacting two-electron system in carbon-nanotube quantum dots. Phys. Rev. Lett. 94, 186806 (2005).

7. Sapmaz, S. et al. Electronic excitation spectrum of metallic carbon nanotubes. Phys. Rev. B 71, 153402 (2005).

8. Cao, J., Wang, Q. \& Dai, H. Electron transport in very clean, as-grown suspended carbon nanotubes. Nature Mater. 4, 745-749 (2005).

9. Makarovski, A., An, L., Liu, J. \& Finkelstein, G. Persistent orbital degeneracy in carbon nanotubes. Phys. Rev. B 74, 155431 (2006).

10. Makarovski, A., Zhukov, A., Liu, J. \& Finkelstein, G. SU(2) and SU(4) Kondo effects in carbon nanotube quantum dots. Phys. Rev. B 75, 241407 (2007).

11. Jarillo-Herrero, P. et al. Electron-hole symmetry in a semiconducting carbon nanotube quantum dot. Nature 429, 389-392 (2004).

12. Ando, T. Spin-orbit interaction in carbon nanotubes. J. Phys. Soc. Jpn. 69, 1757-1763 (2000).
13. Huertas-Hernando, D., Guinea, F. \& Brataas, A. Spin-orbit coupling in curved graphene, fullerenes, nanotubes, and nanotube caps. Phys. Rev. B 74, 155426 (2006).

14. Nowack, K. C., Koppens, F. H. L., Nazarov, Yu V. \& Vandersypen, L. M. K. Coherent control of a single electron spin with electric fields. Science 318, 1430-1433 (2007).

15. Loss, D. \& DiVincenzo, D. P. Quantum computation with quantum dots. Phys. Rev. A. 57, 120-126 (1998).

16. Elzerman, J. M. et al. Single-shot read-out of an individual electron spin in a quantum dot. Nature 430, 431-435 (2004).

17. Petta, J. R. et al. Coherent manipulation of coupled electron spins in semiconductor quantum dots. Science 309, 2180-2184 (2005).

18. Bulaev, D. V., Trauzettel, B. \& Loss, D. Spin-orbit interaction and anomalous spin relaxation in carbon nanotube quantum dots. Preprint at /http://arXiv.org/abs/ 0712.3767) (2007).

19. Trauzettel, B., Bulaev, D. V., Loss, D. \& Burkard, G. Spin qubits in graphene quantum dots. Nature Phys. 3, 192-196 (2007).

20. Awschalom, D. D. \& Flatte, M. E. Challenges for semiconductor spintronics. Nature Phys. 3, 153-159 (2007).

21. Sahoo, S. et al. Electric field control of spin transport. Nature Phys. 1, 99-102 (2005).

22. Tombros, N., van der Molen, S. J. \& van Wees, B. J. Separating spin and charge transport in single-wall carbon nanotubes. Phys. Rev. B 73, 233403 (2006).

23. Tombros, N. et al. Electronic spin transport and spin precession in single graphene layers at room temperature. Nature 448, 571-574 (2007).

24. Minot, E. D., Yaish, Y., Sazonova, V. \& McEuen, P. L. Determination of electron orbital magnetic moments in carbon nanotubes. Nature 428, 536-539 (2004).

25. Cobden, D. H. et al. Spin splitting and even-odd effects in carbon nanotubes. Phys. Rev. Lett. 81, 681-684 (1998).

26. Oreg, Y., Byczuk, K. \& Halperin, B. I. Spin configurations of a carbon nanotube in a nonuniform external potential. Phys. Rev. Lett. 85, 365-368 (2000).

27. Ralchenko, Yu et al. Atomic Spectra Database Version 3.1 .3 (National Institute of Standards and Technology, Gaithersburg, Maryland) (http://physics.nist.gov/ asd3) (accessed, 14 November 2007).

Supplementary Information is linked to the online version of the paper at www.nature.com/nature.

Acknowledgements We thank E. Altman, Y. Gefen, C. L. Henley, Y. Meir, E. Mueller, Y. Oreg, E. I. Rashba, A. Stern and B. Trauzettel for discussions. This work was supported by the NSF through the Center for Nanoscale systems, and by the MARCO Focused Research Center on Materials, Structures and Devices. Samples were fabricated at the Cornell node of the National Nanofabrication Users Network, funded by NSF.

Author Contributions F.K. and S.I. fabricated the devices and performed the experiments. F.K., S.I., D.C.R. and P.L.M. analysed the data and co-wrote the paper. All authors discussed the results and commented on the manuscript.

Author Information Reprints and permissions information is available at www.nature.com/reprints. Correspondence and requests for materials should be addressed to P.L.M. (mceuen@ccmr.cornell.edu). 